\title{
Role of the RAD51 G172T polymorphism in the clinical outcome of cervical cancer patients under concomitant chemoradiotherapy
}

\author{
Augusto Nogueira ${ }^{\mathrm{a}, \mathrm{g}}$, Raquel Catarino ${ }^{\mathrm{a}}$, Ilda Faustino ${ }^{\mathrm{b}}$, Cristina Nogueira-Silva ${ }^{\mathrm{e}}$, Tiago Figueiredo ${ }^{\mathrm{f}}$, \\ Liliana Lombo ${ }^{\mathrm{f}}$, Inês Hilário-Silva ${ }^{\mathrm{a}}$, Deolinda Pereira ${ }^{\mathrm{a}, \mathrm{c}}$, Rui Medeiros ${ }^{\mathrm{a}, \mathrm{c}, \mathrm{d}, \mathrm{g}, *}$ \\ a Molecular Oncology GRP E Virology CI - Portuguese Institute of Oncology, Porto, Portugal \\ ${ }^{\mathrm{b}}$ Medical Oncology Department - Portuguese Institute of Oncology, Porto, Portugal \\ c ICBAS, Abel Salazar Institute for the Biomedical Sciences, Porto, Portugal \\ d CEBIMED, Faculty of Health Sciences, Fernando Pessoa University, Porto, Portugal \\ e Gynaecology/Obstetrics Department, S. Marcos Hospital, Braga, Portugal \\ ${ }^{\mathrm{f}}$ Radiotherapy Department, Portuguese Institute of Oncology, Porto, Portugal \\ ${ }^{g}$ LPCC, Research Department-Portuguese League Against Cancer (NRNorte), Portugal
}

\section{A R T I C L E I N F O}

Article history:

Accepted 17 May 2012

Available online 24 May 2012

\section{Keywords:}

Cervical cancer

RAD51

Polymorphism

DNA repair

Overall survival

\begin{abstract}
A B S T R A C T
Introduction: Cervical cancer is one of the most common cancers diagnosed in women worldwide. Mammalian cells are constantly exposed to a wide variety of genotoxic agents from both endogenous and exogenous sources. The RAD51 protein is required for meiotic and mitotic recombination and plays a central role in homology-dependent recombinational repair of double-strand breaks (DSBs). Given the functional relevance of the DNA repair system on carcinogenesis, potential associations between genetic polymorphisms of DNA repair genes, cancer risk and response to therapy have been intensively evaluated. This is the first study evaluating the role of the RAD51 G172T genetic variants in cancer prognosis and clinical outcome of cervical cancer patients.

Material and methods: We analyzed RAD51 G172T polymorphism genotypes in cervical cancer patients who underwent a platinum-based chemotherapy in combination with radiotherapy. Genotyping was performed by Taqman ${ }^{\mathrm{TM}}$ Allelic Discrimination methodology.

Results and discussion: Concerning the overall survival rates found using Kaplan-Meier method and Log Rank Test, we observed that the mean survival rates were statistically different according to the patients RAD51 genotypes. The group of patients carrying the $\mathrm{T}$ allele present a higher mean survival rate than the other patients (102.3 months vs. 86.4 months, $P=0.020)$. Using the Cox regression analysis, we found an increased overall survival time for T-carrier patients, when compared with GG genotype, with tumor stage, age and presence of lymph nodes as covariates [hazard ratio (HR), $0.373 ; 95 \% \mathrm{CI}, 0.181-0.770 ; P=0.008$ ]. Among patients $(\mathrm{n}=193), R A D 51$ genotype frequency distributions were not under the influence of clinicopathologic characteristics, namely, treatment response $(P=0.508)$, recurrence $(P=0.150)$ and tumor stage $(P=0.250)$. Conclusions: This is the first study evaluating the role of the RAD51 G172T genetic variants in cancer prognosis and clinical outcome of cervical cancer patients. Our results indicate an influence of the RAD51 genetic variants in overall survival of cervical cancer. Thereby, RAD51 G172T genotypes may provide additional prognostic information in cervical cancer patients who underwent cisplatin-based chemotherapy in combination with radiotherapy.
\end{abstract}

(C) 2012 Elsevier B.V. All rights reserved.
Abbreviations: 5-FU, 5-fluorouracil; RCT, radiochemotherapy; HPV, Human papillomavirus; DSB, double-strand break(s); HR, homologous recombination; NHEJ, nonhomologous recombination; kb, kilobase; UTR, untranslated region(s); SNP, single nucleotide polymorphism(s); MAF, minor allele frequency; G, guanine; T, thymine; SD, standard deviation; AJCC, American Joint Committee on Cancer; FIGO, International Federation of Gynaecology and Obstetrics; $\mu$ l, microliter; SPSS, Statistical Package for Social Sciences; P, probability; $\chi^{2}$, Chi-Square; HR, hazard ratio; CI, confidence interval; mRNA, messenger RNA.

* Corresponding author at: IPO, Portuguese Institute of Oncology, Molecular Oncology Group, Laboratórios, Piso 4, Rua Dr. Ant. Bernardino Almeida, 4200-072 Porto, Portugal. Tel.: + 35122 5084000x5414.

E-mail address: ruimedei@ipoporto.min-saude.pt (R. Medeiros).

\section{Introduction}

Cervical carcinoma is the third most common cancer in females, following breast and colorectal cancer. Worldwide, approximately 500,000 new cases are diagnosed each year, with a higher rate of incidence among women of lower socioeconomic status, especially in developing countries (Parkin et al., 2005).

The treatment of cervical cancer has changed over time. Randomized studies (Keys et al., 1999; Morris et al., 1999; Rose et al., 1999) showed that a combined modality approach, including cisplatin with/without 
5-fluorouracil (5-FU) chemotherapy and radiotherapy, improved survival, progression-free survival and recurrence rates in patients with locally advanced cervical cancer. The use of radiotherapy and concurrent cisplatin-based chemotherapy has become the standard treatment in locally advanced cervical cancer (Keys et al., 1999). Surgery and radiation therapy are equally effective for early stage, small-volume disease (Eifel et al., 1991), but for patients with inoperable cervical cancer, radiochemotherapy (RCT) is the preferred treatment.

Human papillomavirus (HPV) is a common sexually transmitted infection and has been shown in epidemiological and molecular studies to be a necessary etiologic agent for cervical cancer (Medeiros et al., 2005; Silva et al., 2011). In considering the factors responsible for the changes seen in HPV and cervical cancer epidemiology, viral factors (increased virulence and predominance of high-risk types), host factors (changes in behavior and immunity), and changes in screening and diagnosis were considered (Barnabas et al., 2006). In addition to HPV infection, smoking is a consistent cofactor for carcinoma of the cervix across retrospective and prospective studies (Matos et al., 2005).

Mammalian cells are constantly exposed to a wide variety of genotoxic agents from both endogenous and exogenous sources. These mutagens sources produce a wide range of DNA lesions as reactive oxygen species, oxidized bases, bulky DNA adducts and DNA strand breaks (Cavalieri et al., 2000; Johnson-Thompson and Guthrie, 2000). The response of the cell to DNA damage and its ability to maintain genomic stability by DNA repair are crucial for preventing cancer initiation and progression (Synowiec et al., 2008).

Double-strand break (DSB) damage is the most injurious lesion observed because it causes cell death or loss of genetic material. Homologous recombination (HR) and non-homologous end joining (NHEJ) are two distinct mechanisms in the repair of double-strand break in mammalian cells (Jara et al., 2007). DNA repair pathways have a critical role for maintaining genomic stability and suppressing mutations (Dixon and Kopras, 2004).

Table 1

Distribution of patient clinicopathologic characteristics.

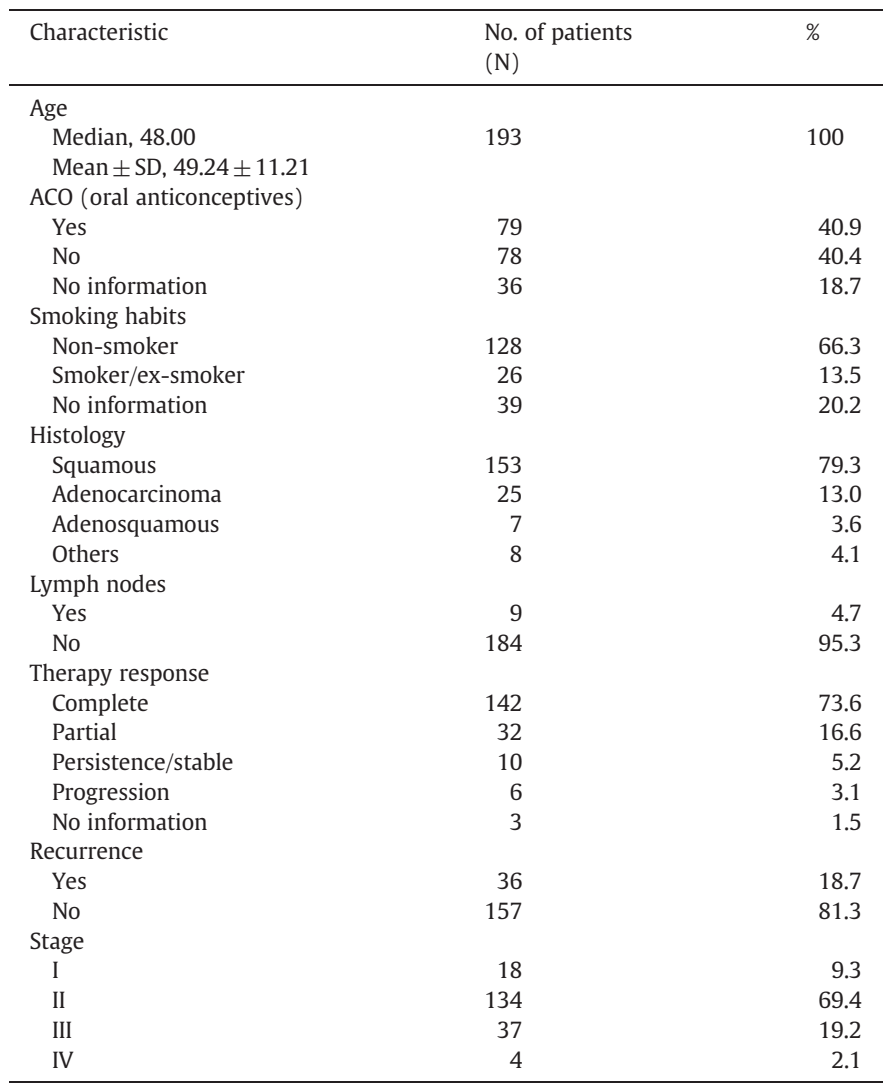

Human RAD51, a homologue of recA protein in Escherichia coli, is required for meiotic and mitotic recombination and plays a central role in homology-dependent recombinational repair of DSBs (Poplawski et al., 2006). In that process, it promotes strand invasion, polymerizing onto a DNA end and mediating the transfer and annealing of the resulting nucleoprotein filament to complementary homologous strand on the intact (Kawabata et al., 2005; Thompson and Schild, 2001).

RAD51 gene is located at chromosome position 15q15.1 in humans (Shinohara et al., 1993). It spans $>39 \mathrm{~kb}$, contains 10 exons and encodes a 339 amino acid protein. The first exon and part of the second exon of RAD51 are located at its 5'-untranslated region (5'-UTR), which may regulate gene expression. RAD51 is highly polymorphic: 143 single nucleotide polymorphisms (SNPs) of RAD51 are known. However, only a few SNPs are located in the coding regions of RAD51, none of which has a minor allele frequency (MAF) $>5 \%$, suggesting that is protein structure may be genetically conservative (Lu et al., 2007).

A single nucleotide polymorphism in the 5'-UTR of the human gene, resulting in a guanine-thymine (G-T) substitution at the 172 position (the RAD51 G172T polymorphism, rs1801321), has been identified. This polymorphism has been reported to be associated with altered gene transcription (Hasselbach et al., 2005). However, no reported studies have investigated the role of RAD51 SNPs in the etiology of cervical cancer. Given the functional relevance of the DNA repair system on carcinogenesis, potential associations between genetic polymorphisms of DNA repair genes, cancer risk and response to therapy have been intensively evaluated (Hung et al., 2005).

The purpose of our study was to assess the effect of RAD51 G172T polymorphism in treatment response in cervical cancer patients.

\section{Materials and methods}

\subsection{Patients}

We conducted a hospital-based study analyzing 193 Caucasian female individuals from the north region of Portugal with cervical cancer, admitted in the Portuguese Institute of Oncology, Porto, Portugal. These patients were randomly selected among those submitted to a chemotherapeutic protocol, between 2000 and 2008, that consisted of platinum-based chemotherapy in combination with radiotherapy. The median age at diagnosis was 48.00 years with a mean age of 49.24 years (standard deviation, $S D=11.21$ ). Patients' clinical characteristics, obtained from medical records, are described in Table 1. The tumor stage was evaluated according to the TNM system proposed by the American Joint Committee on Cancer (AJCC) and the International Federation of Gynaecology and Obstetrics (FIGO), and the assessment of histology type was based the on system of Bethesda classification. Genomic DNA was extracted from peripheral blood samples by using QIAmp DNA Blood Mini kit (QIAGEN), according to the manufacturer's protocol. All samples were obtained with the informed consent of the participants prior to their inclusion in the study, according to Helsinki Declaration principles.

\subsection{Genotyping of RAD51 G172T polymorphism}

Candidate single-nucleotide polymorphism was selected from the best evidence from published studies and through public databases that provide information on the phenotypic risks.

The detection of the RAD51 G172T polymorphism (rs1801321) was carried out essentially as previously described (Rollinson et al., 2007).

The genotyping was performed using Taqman ${ }^{\mathrm{TM}}$ Allelic Discrimination. Primer and probe sequences were as follows: forward primer 5'-CGAGTAGAGAA-GTGGAGCGTAAGC-3', reverse primer 5'-CCGCGCTCCGACTTCA-3', T allele probe 5'-FAM-CGTGCCACtCCCGCGGG-3', G allele probe 5'-VIC-CGTGCC-ACgCCCGCGGG-3'. All allelic discrimination PCR reactions were carried out in $6 \mu \mathrm{l}$ 
volumes using $2.5 \mu \mathrm{l}$ of TaqMan® Universal PCR Master Mix (2X), $0.125 \mu \mathrm{l}$ of $40 \mathrm{x}$ assay mix $2.375 \mu \mathrm{l}$ of sterile $\mathrm{H}_{2} \mathrm{O}$ and $1 \mu \mathrm{l}$ of genomic DNA.

Amplification of DNA was carried out using the following amplification conditions: $95^{\circ} \mathrm{C}$ for $10 \mathrm{~min}$, followed by 45 cycles of $95^{\circ} \mathrm{C}$ for $15 \mathrm{~s}$ and $60^{\circ} \mathrm{C}$ for $1 \mathrm{~min}$. Data capture and analysis was carried thought the ABI 7300 Real Time PCR System (Applied Biosystems) and the Sequence Detection Systems software (Applied Biosystems version 1.2.3).

Quality control included the use of non template controls in all runs and blind replicate genotype assessment on $10 \%$ of the samples. We observed almost complete concordance among duplicates.

\subsection{Statistical analysis}

Analysis of data was performed using the computer software Statistical Package for Social Sciences (SPSS) for Windows (version 17.0). Differences in proportions were evaluated by the $\chi^{2}$ test. The probabilities of survival were calculated and the means life tables were computed using the product-limit estimate of Kaplan-Meier method. The curves were analyzed by the Log Rank (Mantel-Cox) test, a statistical test equality of survival distributions. A level of $P<0.05$ was considered statistically significant. Survival duration was defined as the time between diagnosis and either time of death or time last clinical evaluation of the patient. Cox regression models were used to adjust for potential confounders with RAD51 genotypes fitted as indicator variables.

\section{Results}

We analyzed RAD51 G172T polymorphism genotypes using the Taqman $^{\mathrm{TM}}$ Allelic Discrimination methodology. The frequencies of GG, GT, and TT genotypes were $0.32,0.43$, and 0.25 , respectively.

There were no statistically significant differences in genotype distributions according to the patients clinicopathologic characteristics, namely, treatment response (partial and complete; $P=0.508$ ), recurrence (yes and no; $P=0.150$ ) and tumor stage (I/II and III/IV; $P=0.250$ ) (Table 2).

Concerning the overall survival rates found using Kaplan-Meier method and Log Rank Test (Fig. 1), we observed that the mean survival rates were statistically different according to patients' genotypes. The group of patients carrying the $\mathrm{T}$ allele present a higher mean survival rate than the other patients ( 102.3 months vs. 86.4 months, $P=0.020$ ). Thus, individuals with TT/GT genotypes showed a higher overall survival, conferring a better prognosis for $\mathrm{T}$ allele carrier patients.

Using a multivariate Cox regression model, we found an increased overall survival time for T-carrier patients, when compared with GG genotype, with tumor stage, age and presence of lymph nodes as covariates [hazard ratio (HR), 0.373; 95\% confidence interval (95\% CI), 0.181-0.770; $P=0.008$ ] (Fig. 2).

Table 2

Genotype frequencies of RAD51 G172T polymorphism in cervical cancer patients, according to the clinical characteristics.

\begin{tabular}{llll}
\hline & GG & T carrier & $P^{\mathrm{a}}$ \\
& $\mathrm{N}(\%)$ & $\mathrm{N}(\%)$ & \\
\hline $\begin{array}{l}\text { Therapy response } \\
\text { Not complete }\end{array}$ & $17(8.9)$ & $31(16.3)$ & 0.508 \\
$\quad$ Complete response confirmed & $43(22.6)$ & $99(52.1)$ & \\
Recurrence & & & \\
$\quad$ Yes & $15(7.8)$ & $21(10.9)$ & 0.150 \\
No & $46(23.8)$ & $111(57.5)$ & \\
Stage & & & \\
I/II & $45(23.3)$ & $107(55.4)$ & 0.250 \\
III/IV & $16(8.3)$ & $25(13.0)$ & \\
\hline
\end{tabular}

a Chi square test.

b Parcial + persistence + progression + no response.

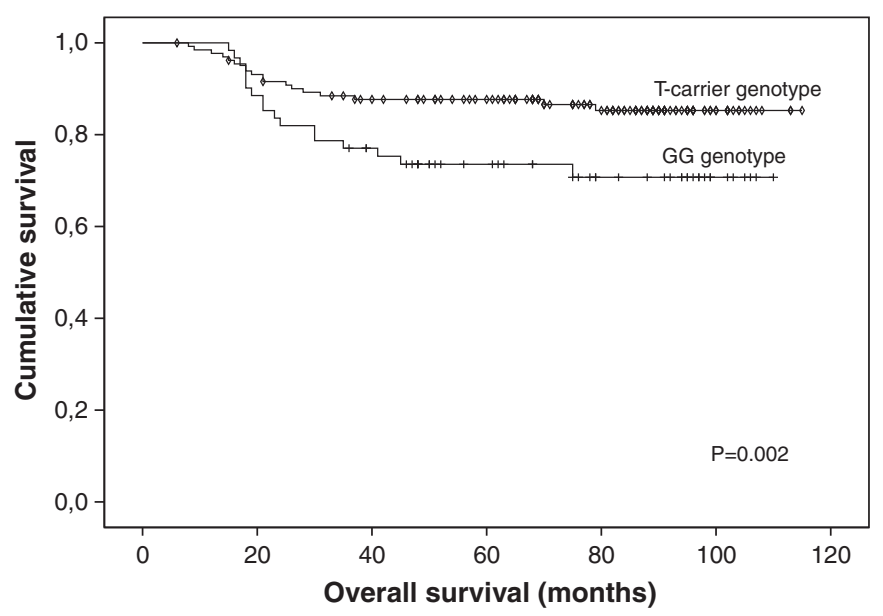

Fig. 1. Overall survival by Kaplan-Meier and Log Rank Test of cervical cancer patients, according to RAD51 G172T genotypes.

\section{Discussion}

Molecular epidemiology studies have demonstrated that the SNPs were found in nearly all human DNA repair genes that have been investigated so far (Mohrenweiser et al., 2003), and some of them were shown modulate levels of DNA damage, individual DNA repair capacity and cancer risk (Goode et al., 2002; Matullo et al., 2003). There is increasing evidence that reduced DNA repair capacity resulting from genetic polymorphisms of various DNA repair genes is associated with improved survival with platinum-based chemotherapy (Gurubhagavatula et al., 2004; Ryu et al., 2004; Zhou et al., 2004). Thus, DNA repair capacity is considered both a barrier to carcinogenesis and a crucial molecular pathway implicated in the resistance to cisplatin-based chemotherapy (Kelland, 2007; Wei et al., 2000). Therefore, gene polymorphisms of DNA repair factors are therefore obvious candidates for determinants of repair capacity and chemotherapy efficacy (Sancar, 1995). Moreover, the functional variants caused by SNPs in genes encoding drugmetabolizing enzymes, transporters, ion channels, drug receptors, cell cycle proteins and DNA repair are known to be associated with individual variation in survival, drug responses, and toxicity (Henson et al., 2006; Costa et al., 2008; Nogueira et al., 2010; Catarino et al., 2008; Craveiro et al., 2012; Santos et al., 2006; Medeiros et al., 2003).

Faithful genome transmission requires the co-ordination of a network of pathways such as cell cycle checkpoint, DNA replication, DNA

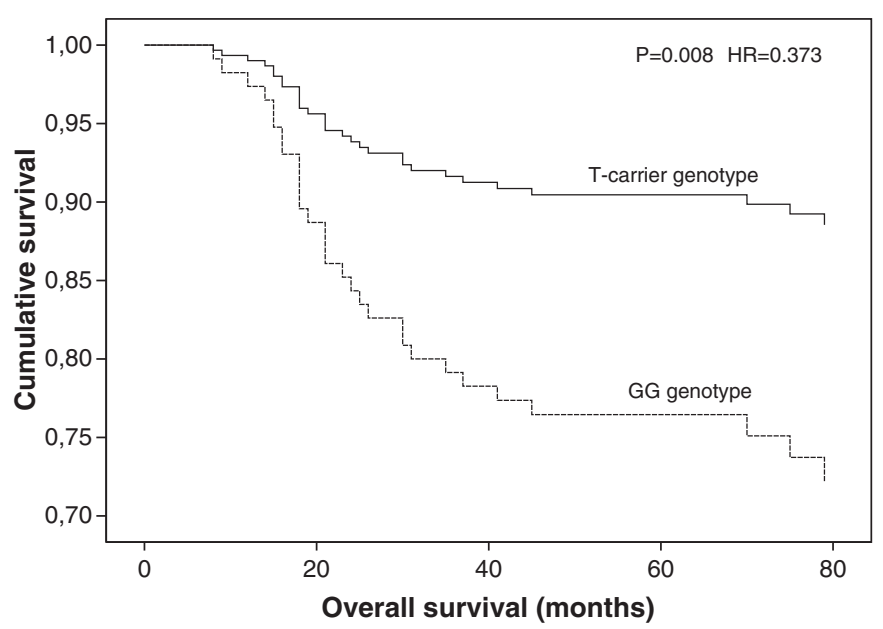

Fig. 2. Cox regression analysis of overall survival adjusted with stage, age and presence of lymph nodes as covariates, according to the RAD51 G172T polymorphism. 
repair/recombination and programmed cell death (Daboussi et al., 2002). The RAD51 gene is an essential factor for homologous recombination and the repair of DNA DSBs, binding transiently to both single stranded and double stranded DNA during the recombination reaction. RAD51 has been found to be increased in expression in a wide range of human tumors, most likely contributing to chemotherapeutic resistance of these tumors (Klein, 2008). According to previous studies in other tumor models, the study of RAD51 gene seems to be important for the prediction of treatment response with chemotherapeutic drugs (Connell et al., 2006). It remains unclear whether the link of RAD51 expression to sensitivity to platinum agents may affects the prognosis (Takenaka et al., 2007).

In previous studies, RAD51 expression was identified as an independent predictor for tumor recurrence, as well as for tumor progression. These data demonstrate RAD51 expression as a clinical significant prognostic marker. Increased RAD51 protein levels could lead to uncontrolled recombination, genome instability, prevention of apoptosis, cell cycle arrest and increased resistance tumor to radiotherapy and chemotherapy. Down-regulation of RAD51 protein by anti-sense oligonucleotides or small molecules that sensitizes cells to DNA-damaging agents (Ohnishi et al., 1998) may be a promising strategy for tumor therapy (Henning and Sturzbecher, 2003).

Polymorphisms in UTR region have been shown to be important for cell proliferation, differentiation, tumor suppression, and metastasis suppression on several genes (Facher et al., 1997; Fan et al., 1996). For this reason, RAD51 G172T polymorphism at the 5'-UTR region can have an important role on protein expression and stability. As it is located in the $5^{\prime}$-UTR region of the RAD51 gene, this genetic variation could affect mRNA stability or translational efficiency, leading to altered polypeptide product levels and altering the function of the final product the RAD51 protein (Hasselbach et al., 2005; Lu et al., 2007).

Some studies showed an association between the RAD51 G172T polymorphism and risk of breast (Kuschel et al., 2002; Lee et al., 2005), epithelial ovarian cancers (Auranen et al., 2005), and squamous cell carcinoma of the head and neck (Lu et al., 2007). However, none of these studies investigated the role of this polymorphism in the therapy response as a prognostic factor to cervical cancer patients. In this study, our results indicate an influence of the RAD51 genetic variants in overall survival of cervical cancer. Thereby, we suggest that the RAD51 G172T genotypes may provide additional prognostic information in cervical cancer patients who underwent cisplatin-based chemotherapy in combination with radiotherapy. Our results indicate that individuals carrying the $\mathrm{T}$ allele present an overall survival of approximately 102 months after chemotherapy, compared with 86 months for individuals carrying the GG genotype.

The functional consequences of $R A D 51$ polymorphisms are not fully understood. Although future functional studies are needed to clarify this question, we hypothesized that the RAD51 variant alleles may originate a lower activity of RAD51 promoter (Nogueira et al., 2010). Thus, individuals carrying the RAD51 $172 T T$ genotype may have a lower cellular capacity for DSB repair or present a greater sensitivity to chemotherapeutic agents than carriers of other genotypes, supporting our observation that the RAD51 172TT genotype has a protective effect on clinical outcome of cervical cancer patients.

The positive effect in treatment response that we observe for RAD51 G172T polymorphism suggests that it may be associated with decreasing RAD51 expression levels in tumor cells, originating sensitized tumor cells with lower cellular capacity for damage repair and most sensitizes for chemotherapeutic agents, thus increased the efficacy of chemotherapy and giving a better prognosis for cervical cancer patients. Although some known regulatory elements are associated with 5'-UTRs (Gray and Wickens, 1998; van der Velden and Thomas, 1999), it is not clear how $172 \mathrm{~T}$ polymorphism might affect gene function. Further studies may clarify these putative functional implications.

To the best of our knowledge, this is the first study evaluating the role of the RAD51 G172T genetic variants in cancer prognosis and clinical outcome of cervical cancer patients suggesting a role as a molecular marker for predicting the clinical outcome of cervical cancer patients. Patient pharmacogenomic profiling is being used to predict whether the selected chemotherapy will be really effective and tolerable to the patient. Thus, DNA repair genotypes may be add to the pharmacogenomic strategy to elucidate how an adversely or favorably response to chemotherapy outcome may occur.

\section{Conflict of interest}

All of the authors declare no conflict of interest that could inappropriately influence this work.

\section{Acknowledgments}

The authors thank the Research Department-Portuguese League Against Cancer (NRNorte) for their support. We acknowledge funding of this work by the Ministry of Health of Portugal. We also gratefully acknowledge the financial support of an individual grant for Post Doc project of Raquel Catarino from the Minister of Science, Technology and Superior Education-FCT (Fundação para a Ciência e Tecnologia: SFRH/BPD/72932/2010).

\section{References}

Parkin, D.M., et al., 2005. Global cancer statistics, 2002. CA Cancer J. Clin. 55 (2), 74-108.

Keys, H.M., et al., 1999. Cisplatin, radiation, and adjuvant hysterectomy compared with radiation and adjuvant hysterectomy for bulky stage IB cervical carcinoma. N. Engl. J. Med. 340 (15), 1154-1161.

Morris, M., et al., 1999. Pelvic radiation with concurrent chemotherapy compared with pelvic and para-aortic radiation for high-risk cervical cancer. N. Engl. J. Med. 340 (15), 1137-1143.

Rose, P.G., et al., 1999. Concurrent cisplatin-based radiotherapy and chemotherapy for locally advanced cervical cancer. N. Engl. J. Med. 340 (15), 1144-1153.

Eifel, P.J., et al., 1991. Early stage I adenocarcinoma of the uterine cervix: treatment results in patients with tumors less than or equal to $4 \mathrm{~cm}$ in diameter. Gynecol. Oncol. 41 (3), 199-205.

Silva, J., et al., 2011. Oncogenic HPV types infection in adolescents and university women from north Portugal: from self-sampling to cancer prevention. J. Oncol. 953469 .

Medeiros, R., et al., 2005. Characterization of HPV genotype profile in squamous cervical lesions in Portugal, a southern European population at high risk of cervical cancer. Eur. J. Cancer Prev. 14 (5), 467-471.

Barnabas, R.V., et al., 2006. Epidemiology of HPV 16 and cervical cancer in Finland and the potential impact of vaccination: mathematical modelling analyses. PLoS Med. 3 (5), e138.

Matos, A., et al., 2005. The influence of smoking and other cofactors on the time to onset to cervical cancer in a southern European population. Eur. J. Cancer Prev. 14 (5), 485-491.

Cavalieri, E., et al., 2000. Estrogens as endogenous genotoxic agents-DNA adducts and mutations. J. Natl. Cancer Inst. Monogr. 27, 75-93.

Johnson-Thompson, M.C., Guthrie, J., 2000. Ongoing research to identify environmental risk factors in breast carcinoma. Cancer 88 (5 Suppl.), 1224-1229.

Synowiec, E., et al., 2008. Association between DNA damage, DNA repair genes variability and clinical characteristics in breast cancer patients. Mutat. Res. 648 (1-2), 65-72.

Jara, L., et al., 2007. RAD51 135G >C polymorphism and risk of familial breast cancer in a South American population. Cancer Genet. Cytogenet. 178 (1), 65-69.

Dixon, K., Kopras, E., 2004. Genetic alterations and DNA repair in human carcinogenesis. Semin. Cancer Biol. 14 (6), 441-448.

Poplawski, T., et al., 2006. DNA damage and repair in gastric cancer-a correlation with the hOGG1 and RAD51 genes polymorphisms. Mutat. Res. 601 (1-2), 83-91.

Kawabata, M., Kawabata, T., Nishibori, M., 2005. Role of recA/RAD51 family proteins in mammals. Acta Med. Okayama 59 (1), 1-9.

Thompson, L.H., Schild, D., 2001. Homologous recombinational repair of DNA ensures mammalian chromosome stability. Mutat. Res. 477 (1-2), 131-153.

Shinohara, A., et al., 1993. Cloning of human, mouse and fission yeast recombination genes homologous to RAD51 and recA. Nat. Genet. 4 (3), 239-243.

Lu, J., et al., 2007. $172 \mathrm{G}>\mathrm{T}$ variant in the $5^{\prime}$ untranslated region of DNA repair gene RAD51 reduces risk of squamous cell carcinoma of the head and neck and interacts with a P53 codon 72 variant. Carcinogenesis 28 (5), 988-994.

Hasselbach, L., et al., 2005. Characterisation of the promoter region of the human DNArepair gene Rad51. Eur. J. Gynaecol. Oncol. 26 (6), 589-598.

Hung, R.J., et al., 2005. Genetic polymorphisms in the base excision repair pathway and cancer risk: a HuGE review. Am. J. Epidemiol. 162 (10), 925-942.

Rollinson, S., et al., 2007. RAD51 homologous recombination repair gene haplotypes and risk of acute myeloid leukaemia. Leuk. Res. 31 (2), 169-174.

Mohrenweiser, H.W., Wilson III, D.M., Jones, I.M., 2003. Challenges and complexities in estimating both the functional impact and the disease risk associated with the 
extensive genetic variation in human DNA repair genes. Mutat. Res. 526 (1-2), 93-125.

Goode, E.L., Ulrich, C.M., Potter, J.D., 2002. Polymorphisms in DNA repair genes and associations with cancer risk. Cancer Epidemiol. Biomarkers Prev. 11 (12), 1513-1530.

Matullo, G., et al., 2003. Combination of DNA repair gene single nucleotide polymorphisms and increased levels of DNA adducts in a population-based study. Cancer Epidemiol. Biomarkers Prev. 12 (7), 674-677.

Gurubhagavatula, S., et al., 2004. XPD and XRCC1 genetic polymorphisms are prognostic factors in advanced non-small-cell lung cancer patients treated with platinum chemotherapy. J. Clin. Oncol. 22 (13), 2594-2601.

Zhou, W., et al., 2004. Excision repair cross-complementation group 1 polymorphism predicts overall survival in advanced non-small cell lung cancer patients treated with platinum-based chemotherapy. Clin. Cancer Res. 10 (15), 4939-4943.

Ryu, J.S., et al., 2004. Association between polymorphisms of ERCC1 and XPD and survival in non-small-cell lung cancer patients treated with cisplatin combination chemotherapy. Lung Cancer 44 (3), 311-316.

Kelland, L., 2007. The resurgence of platinum-based cancer chemotherapy. Nat. Rev. Cancer 7 (8), 573-584.

Wei, Q., Frazier, M.L., Levin, B., 2000. DNA repair: a double-edged sword. J. Natl. Cancer Inst. 92 (6), 440-441.

Sancar, A., 1995. DNA repair in humans. Annu. Rev. Genet. 29, 69-105.

Henson, S.E., et al., 2006. Pir51, a Rad51-interacting protein with high expression in aggressive lymphoma, controls mitomycin C sensitivity and prevents chromosomal breaks. Mutat. Res. 601 (1-2), 113-124.

Costa, S., et al., 2008. XRCC1 Arg399Gln and RAD51 5'UTR G135C polymorphisms and their outcome in tumor aggressiveness and survival of Portuguese breast cancer patients. Breast Cancer Res. Treat. 109 (1), 183-185.

Nogueira, A., et al., 2010. Influence of DNA repair RAD51 gene variants in overall survival of non-small cell lung cancer patients treated with first line chemotherapy. Cancer Chemother. Pharmacol. 66 (3), 501-506.

Catarino, R., et al., 2008. Oncogenic virus-associated neoplasia: a role for cyclin D1 genotypes influencing the age of onset of disease? Biochem. Biophys. Res. Commun. 370 (1), 118-122.

Craveiro, R., et al., 2012. The Role of p73 G4C14-to-A4T14 Polymorphism in the Susceptibility to Cervical Cancer. DNA Cell Biol. 31 (2), 224-229.
Santos, A.M., et al., 2006. Linking TP53 codon 72 and P21 nt590 genotypes to the development of cervical and ovarian cancer. Eur. J. Cancer 42 (7), 958-963.

Medeiros, R., et al., 2003. Platinum/paclitaxel-based chemotherapy in advanced ovarian carcinoma: glutathione S-transferase genetic polymorphisms as predictive biomarkers of disease outcome. Int. J. Clin. Oncol. 8 (3), 156-161.

Daboussi, F., et al., 2002. DNA double-strand break repair signalling: the case of RAD51 post-translational regulation. Cell. Signal. 14 (12), 969-975.

Klein, H.L., 2008. The consequences of Rad51 overexpression for normal and tumor cells. DNA Repair (Amst.) 7 (5), 686-693.

Connell, P.P., et al., 2006. Pilot study examining tumor expression of RAD51 and clinical outcomes in human head cancers. Int. J. Oncol. 28 (5), 1113-1119.

Takenaka, T., et al., 2007. Combined evaluation of Rad51 and ERCC1 expressions for sensitivity to platinum agents in non-small cell lung cancer. Int. J. Cancer 121 (4), 895-900.

Ohnishi, T., et al., 1998. In vitro and in vivo potentiation of radiosensitivity of malignant gliomas by antisense inhibition of the RAD51 gene. Biochem. Biophys. Res. Commun. 245 (2), 319-324.

Henning, W., Sturzbecher, H.W., 2003. Homologous recombination and cell cycle checkpoints: Rad51 in tumour progression and therapy resistance. Toxicology 193 (1-2), 91-109.

Facher, E.A., et al., 1997. Association between human cancer and two polymorphisms occurring together in the p21Waf1/Cip1 cyclin-dependent kinase inhibitor gene. Cancer 79 (12), 2424-2429.

Fan, H., et al., 1996. Suppression of malignancy by the 3' untranslated regions of ribonucleotide reductase R1 and R2 messenger RNAs. Cancer Res. 56 (19), 4366-4369.

Kuschel, B., et al., 2002. Variants in DNA double-strand break repair genes and breast cancer susceptibility. Hum. Mol. Genet. 11 (12), 1399-1407.

Lee, K.M., et al., 2005. Genetic polymorphisms of selected DNA repair genes, estrogen and progesterone receptor status, and breast cancer risk. Clin. Cancer Res. 11 (12), 4620-4626.

Auranen, A., et al., 2005. Polymorphisms in DNA repair genes and epithelial ovarian cancer risk. Int. J. Cancer 117 (4), 611-618.

Gray, N.K., Wickens, M., 1998. Control of translation initiation in animals. Annu. Rev. Cell Dev. Biol. 14, 399-458.

van der Velden, A.W., Thomas, A.A., 1999. The role of the $5^{\prime}$ untranslated region of an mRNA in translation regulation during development. Int. J. Biochem. Cell Biol. 31 (1), 87-106. 